\title{
Bit and Power Loading for OFDM with An Amplify-and-Forward Cooperative Relay
}

\author{
$\mathrm{Na}$ Yi, Yi Ma, and Rahim Tafazolli \\ Centre for Communication Systems Research \\ University of Surrey, UK, GU2 7XH. \\ Emails:\{n.yi, y.ma, r.tafazolli\}@surrey.ac.uk
}

\begin{abstract}
In this paper, we propose a rate-adaptive bit and power loading approach for the OFDM-based relaying communications. The cooperative relay operates in the half-duplex amplify-and-forward mode. The source and the relay has the separate power constraints. The maximum-ratio combining is employed at the destination for maximizing the received SNR. Assuming the perfect channel knowledge available at all nodes, the proposed approach is to maximize the throughput (the number of bits/symbol) at the given power constraint and the target link performance. Unlike the water-filling method, the proposed approach does not need the iterative loading process, and can offer the sub-optimum performance. Computer simulations are used to test the proposed approach for various scenarios with respect to the relay location or the distributed power allocation.
\end{abstract}

\section{INTRODUCTION}

Since 1971, van der Mulen introduced the three-terminal relay channel [1], there has been already a significant research activity towards what is called cooperative communications. These have included the work on capacity theorem (e.g., [2][3]), relaying or cooperation protocols (e.g., [4]-[5]), diversity and multiplexing gain (e.g., [6]-[7]), etc. Recently, European Commission has been considering to apply the cooperative relay concept in the future high-data-rate wireless services. The relaying node can either be a fixed relaying station or a low-mobility user [8]. The aim is to combine the merits of the cooperative relay with the advance air-interface such as orthogonal frequency-division multiplexing (OFDM). Then, one of interesting research issues can be the bit and power loading (BPL) for the OFDM-based relaying communication.

The state-of-the-art BPL approaches can be classified into two categories [9], i.e., the water-filling approach and the sub-optimum approach. Although the fore one can offer the optimum solution, it can cause the fractional number of bits/symbol. This can significantly increase the implementation complexity for encoder/decoder (or modulation/demodulation). Practically, we can employ the suboptimum approach such as margin-adaptive or rate-adaptive to offer the near water-filling performance. In 1999, Wong et al. have intensively investigated the margin-adaptive approach for the OFDM system [10]. Their investigation has included the single-user link adaption and the multi-user resource competition. Their objective was to minimize the power cost per bit at a fixed data rate and a target symbol-errorrate (SER). Recently, we have proposed the margin-adaptive approach for the OFDM-based three-node relaying commu- nications [11]. The proposed approach could offer the nearoptimum performance when the total transmit-power could be optimally allocated among the distributed senders. Although the optimum distributed power allocation is reasonable for many communication systems (e.g., [12]-[13]), it may not be a good assumption while the distributed senders have their separate power constraints. In this scenario, the water-filling approach needs the iterative resource allocation process [14].

In this paper, we propose a rate-adaptive approach for the OFDM-based three-node relaying communication. Both the source and the relay have their separate power constraints. The cooperative relay operates in the half-duplex amplify-andforward (AF) mode. The maximum-ratio combining (MRC) is employed at the destination for maximizing the received signal-to-noise ratio (SNR). Assuming the channel side information perfectly available at all nodes, the proposed BPL approach is to maximize the number of bits per symbol at the target SER performance. Basically, our approach has two steps. The first step is called the optimum loading process, for which the BPL should fulfill the optimum resource allocation criterion derived in [11]. While either the source or the relay reaches its own power constraint, the proposed approach can start the second step called the compensation process. This process is based on our newly established constrained optimization criterion. Moreover, computer simulations are used to evaluate the proposed approach for various system setups with respect to the relay location or the distributed power allocation.

\section{SySTEM DESCRIPTION}

\section{A. OFDM With Single AF Relay}

OFDM has received many applications for high-data-rate communications. A comprehensive survey of OFDM can be found in [15]. The basic idea is to transmit the informationbearing symbols over a number of low-rate orthogonal subcarriers. This can be implemented by employing the IDFT at the transmitter and the DFT at the receiver. A cyclic prefix can be employed for introducing the channel circulant property and mitigating the inter-block interference.

Fig. 1 depicts the block diagram of the considered OFDM system, where a half-duplex AF relay is employed to help the source-to-destination (SD) link communication. Prior to transmission, the information-bearing bits are first fed into the BPL component to produce an $M \times 1$ symbol 


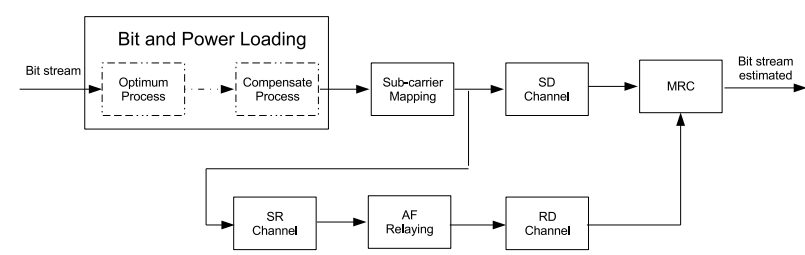

Fig. 1. Block diagram of OFDM systems with single AF cooperative relay

block $c^{(\mathrm{s})}=\left[c_{0}^{(\mathrm{s})}, c_{1}^{(\mathrm{s})}, \cdots, c_{M-1}^{(\mathrm{s})}\right]$, where $c^{(\mathrm{s})}$ denotes the information-bearing symbol (with the variance $\sigma_{c}^{2}=1$ ) or zero symbol sent by the source. Then, these symbols are mapped onto $M$ subcarriers with the transmit power $\tilde{\boldsymbol{p}}^{(\mathrm{s})}=$ $\left[\tilde{p}_{0}^{(\mathrm{s})}, \tilde{p}_{1}^{(\mathrm{s})}, \cdots, \tilde{p}_{M-1}^{(\mathrm{s})}\right]$, where $\tilde{p}_{m}^{(\mathrm{s})}$ denotes the source power for the $m$ th subcarrier. This block goes through the SD channel and the source-to-relay (SR) channel, respectively. Denoting $h$ to be the frequency-domain channel coefficient, the received symbol on the $m$ th subcarrier (at the destination or the relay) is expressible as

$$
\begin{array}{ll}
\text { SD link : } & y_{m}^{(\mathrm{sd})}=\sqrt{\tilde{p}_{m}^{(\mathrm{s})}} h_{m}^{(\mathrm{sd})} c_{m}^{(\mathrm{s})}+v_{m}^{(\mathrm{sd})}, \\
\text { SR link : } & y_{m}^{(\mathrm{sr})}=\sqrt{\tilde{p}_{m}^{(\mathrm{s})}} h_{m}^{(\mathrm{sr})} c_{m}^{(\mathrm{s})}+v_{m}^{(\mathrm{sr})},
\end{array}
$$

where $v$ is the Gaussian noise with zero mean and variance $\mathcal{N}_{o}$, and the superscript ${ }^{(\mathrm{sd})},{ }^{(\mathrm{sr})}$ is the label for the SD channel and the SR channel. The AF relay can weight each received symbol with the coefficient $\sqrt{\beta_{m}}$, and transmit them through the relay-to-destination (RD) channel. Then, the destination receives another version of the information as below

$$
\begin{aligned}
y_{m}^{(\mathrm{rd})} & =h_{m}^{(\mathrm{rd})}\left(\sqrt{\beta_{m}} y_{m}^{(\mathrm{sr})}\right)+v_{m}^{(\mathrm{rd})}, \\
& =h_{m}^{(\mathrm{rd})} \sqrt{\beta_{m}} \sqrt{\tilde{p}_{m}^{(\mathrm{s})}} h^{(\mathrm{sr})} c_{m}^{(\mathrm{s})}+h_{m}^{(\mathrm{rd})} \sqrt{\beta_{m}} v_{m}^{(\mathrm{sr})}+v_{m}^{(\mathrm{rd})},
\end{aligned}
$$

Then, the following operation is performed on $y_{m}^{(\mathrm{rd})}$ to whiten the noise variance to $\mathcal{N}_{o}$

$$
\bar{y}_{m}^{(\mathrm{rd})}=\underbrace{\left(\left|h_{m}^{(\mathrm{rd})} \sqrt{\beta_{m}}\right|^{2}+1\right)^{-\frac{1}{2}}}_{\theta_{m}} y_{m}^{(\mathrm{rd})} .
$$

The destination can estimate the symbol from the MRC of $y_{m}^{\text {(sd) }}$ and $\bar{y}_{m}^{(\mathrm{rd})}$. In AF relaying communications, the instant finalSER $\mathcal{P}_{m}^{(\mathrm{mrc})}$ for each subcarrier is only related to the effective SNR after the MRC, which can be obtained as below

$$
\begin{aligned}
\gamma_{m}^{(\mathrm{mrc})} & =\frac{\left(\left|h_{m}^{(\mathrm{sd})}\right|^{2}+\left|\theta_{m} h_{m}^{(\mathrm{rd})} \sqrt{\beta_{m}} h_{m}^{(\mathrm{sr})}\right|^{2}\right) \tilde{p}_{m}^{(\mathrm{s})}}{\mathcal{N}_{o}}, \\
& =\frac{\tilde{p}_{m}^{(\mathrm{s})}}{\mathcal{N}_{o}}\left(\left|h_{m}^{(\mathrm{sd})}\right|^{2}+\frac{\left|h_{m}^{(\mathrm{sr})}\right|^{2}\left|h_{m}^{(\mathrm{rd})}\right|^{2} \beta_{m}}{\beta_{m}\left|h_{m}^{(\mathrm{rd})}\right|^{2}+1}\right) .
\end{aligned}
$$

The relay power for the $m$ th subcarrier is expressible as

$$
\tilde{p}_{m}^{(\mathrm{r})}=\beta_{m}\left(\left|h_{m}^{(\mathrm{sr})}\right|^{2} \tilde{p}_{m}^{(\mathrm{s})}+\mathcal{N}_{o}\right) .
$$

Moreover, the source and the relay have the following power constraint

$$
P=\sum_{m=0}^{M-1} \tilde{p}_{m}^{(\mathrm{s})}, \quad Q=\sum_{m=0}^{M-1} \tilde{p}_{m}^{(\mathrm{r})} .
$$

The proposed BPL approach is to maximize the number of bits/symbol under separate power constraints for the target SNR $\gamma^{(\mathrm{d})}$ which is corresponding to the target SER $\mathcal{P}^{(\mathrm{d})}$

$$
\max _{P, Q} B=\sum_{m=0}^{M-1} b_{m},
$$

where $b_{m}$ is the amount of bits on the $m$ th subcarrier.

\section{B. BPL for OFDM based end-to-end communication}

Here, we introduce briefly the rate-adaptive algorithm for the single-antenna single-link OFDM communication. The basic structure can be described as below.

Consider $\mathcal{P}^{\text {(d) }}$ to be the target SER on the $m$ th subcarrier. Allocating the amount of $b_{m}$ bits onto the $m$ th subcarrier needs the power $p_{m}$ as below [10]

$$
p_{m}=\frac{\mathcal{N}_{o}}{3\left|h_{m}\right|^{2}}\left[\mathcal{Q}^{-1}\left(\mathcal{P}^{(\mathrm{d})} / 4\right)\right]^{2}\left(2^{b_{m}}-1\right),
$$

where $\mathcal{Q}(\cdot)$ is the Gaussian Q-function.

Given the power constraint $P$, the rate-adaptive algorithm is to maximize the Lagrangian

$$
\mathcal{L}=\sum_{m=0}^{M-1} b_{m}+\lambda\left(\sum_{m=0}^{M-1} p_{m}-P\right),
$$

where $\lambda$ is the Lagrangian multiplier for the constraint $\sum_{m=0}^{M-1} p_{m}-P=0$. It is observed that $b_{m}$ is a convex and increasing function of $p_{m}$. Thus, (12) leads to the following BPL algorithm

1) Initialization:

For all $m$, let $i=0$ (iteration index), $b_{m}=0, p_{0, m}=0$, $\Delta_{p_{i, m}}=p_{i, m}-p_{i-1, m}$

2) Bit-power iterations:

$i=i+1$;

$\hat{m}=\arg \min _{m} \Delta_{p_{i-1, m}}$;

$p_{i, \hat{m}}=p_{i-1, m}+\Delta_{p_{i, \hat{m}}}$;

$b_{\hat{m}}=b_{\hat{m}}+1$;

$\tilde{P}_{i}=\sum_{m=0}^{M-1} p_{i, m}$;

Repeat Step 2) when $\tilde{P}_{i}<P$;

End

\section{BPL FOR ThE RELAYING COMMUNICATION}

In this section, we will introduce the proposed BPL approach for the AF relaying protocol. Basically, this approach can be implemented as two steps. At the first step, the bit and power can be optimally allocated onto each subcarrier according to the resource allocation criterion established in [11]. While either the source or the relay reaches its own power constraint, the BPL approach can start the second step, i.e., the compensation process. The second step stops while the separate power constraint is fulfilled. 


\section{Step 1: Optimum Loading Process}

This step is based on the optimum resource allocation criterion for the multi-channel AF relaying communications in [11]. The objective is to allocate bits onto the desired subcarrier, which needs the minimum total power consumption $\tilde{P}_{m}=\tilde{p}_{m}^{(\mathrm{s})}+\tilde{p}_{m}^{(\mathrm{r})}$. As a summary, we can first define a number of coefficients as below

$$
\begin{gathered}
\mathrm{A}_{m}=\left|h_{m}^{(\mathrm{sd})}\right|^{2}\left|h_{m}^{(\mathrm{sr})}\right|^{2}-\left|h_{m}^{(\mathrm{sd})}\right|^{2}\left|h_{m}^{(\mathrm{rd})}\right|^{2}-\left|h_{m}^{(\mathrm{sr})}\right|^{2}\left|h_{m}^{(\mathrm{rd})}\right|^{2} \cdot \\
\mathrm{D}_{m}=\left|h_{m}^{(\mathrm{sd})}\right|^{2}\left|h_{m}^{(\mathrm{rd})}\right|^{2} \tilde{P}_{m}+\left|h_{m}^{(\mathrm{sd})}\right|^{2} \mathcal{N}_{o}+\left|h_{m}^{(\mathrm{sr})}\right|^{2}\left|h_{m}^{(\mathrm{rd})}\right|^{2} \tilde{P}_{m} . \\
\mathrm{B}_{m}=\left|h_{m}^{(\mathrm{sr})}\right|^{2}-\left|h_{m}^{(\mathrm{rd})}\right|^{2} . \\
\mathrm{C}_{m}=\left|h_{m}^{(\mathrm{sd})}\right|^{2} \tilde{P}_{m}+\mathcal{N}_{o} \\
\Delta_{m}=4 \mathrm{~A}_{m}^{2} \mathrm{C}_{m}^{2}-4 \mathrm{~A}_{m} \mathrm{~B}_{m} \mathrm{C}_{m} \mathrm{D}_{m} .
\end{gathered}
$$

Then, we can have the following result.

Theorem. 1: Define

$$
\begin{gathered}
\phi=\frac{-2 \mathrm{~A}_{m} \mathrm{C}_{m}-\sqrt{\Delta_{m}}}{2 \mathrm{~A}_{m} \mathrm{~B}_{m}} \\
\phi_{t}=\frac{\left|h_{m}^{(\mathrm{sd})}\right|^{2} \mathcal{N}_{o}}{\left|h_{m}^{(\mathrm{sr})}\right|^{2}\left(\left|h_{m}^{(\mathrm{rd})}\right|^{2}-\left|h_{m}^{(\mathrm{sd})}\right|^{2}\right)} .
\end{gathered}
$$

Given the target SNR $\gamma^{(\mathrm{d})}$, the source power should be $\tilde{p}_{m}^{(\mathrm{s})}=$ $\phi$ under the conditions: C1) $\left|h_{m}^{(\text {sd) }}\right|<\left|h_{m}^{(\text {rd })}\right|$, and C2) $\tilde{P}_{m} \in$ $\left(\phi_{t},\left(\gamma^{(\mathrm{d})} \mathcal{N}_{o}\right) /\left(\left|h_{m}^{(\mathrm{sd})}\right|^{2}\right)\right)$. Otherwise, the source power should be $\left.\tilde{p}_{m}^{(\mathrm{s})}=\tilde{P}_{m}=\left(\gamma^{(\mathrm{d})} \mathcal{N}_{o}\right) /\left(\left|h_{m}^{(\mathrm{sd})}\right|^{2}\right)\right)$.

Then, this optimum loading process can be implemented as follows:

S1: Calculating the target SNR $\gamma^{(\mathrm{d})}$, i.e., $\gamma^{(\mathrm{d})}=1 / 3\left[\mathcal{Q}^{-1}\left(\mathcal{P}^{(\mathrm{d})} / 4\right)\right]^{2}\left(2^{b_{m}}-1\right)$;

S2: $\quad$ Calculating the range of $\tilde{P}_{m}$ in $\mathrm{C} 2$ );

S3: If the conditions $\mathrm{C} 1$ ) and $\mathrm{C} 2$ ) hold, let $\tilde{p}_{m}^{(\mathrm{s})}=\phi$. Otherwise, let $\left.\tilde{p}_{m}^{(\mathrm{s})}=\tilde{P}_{m}=\left(\gamma^{(\mathrm{d})} \mathcal{N}_{o}\right) /\left(\left|h_{m}^{(\mathrm{sd})}\right|^{2}\right)\right)$ and go to $\mathrm{S} 5$;

S4: $\quad$ Searching $\tilde{P}_{m}$ over the range in C2) to minimize $\left|\gamma_{m}^{(\mathrm{mrc})}-\gamma^{(\mathrm{d})}\right|$;

S5: Load bit onto the desired subcarrier with the minimum $\tilde{P}_{m}$ (This step can follow the BPL algorithm addressed in Section II-B).

End

The above BPL process can minimize the power consumption per bit. While the distributed senders do not have the separate power constraint, this process can offer the optimum distributed power allocation. On the other hand, Theorem 1 shows that the relay should re-transmit the received symbols only for the conditions $\mathrm{C} 1$ )-C2). This can result in unbalance power allocation between the source and the relay. For example, while the source power has been used up, the relay may still has plenty of power need to be allocated. This is a waste of relay resource. Hence, we propose the following compensation process.

\section{Step 2: Compensation Process}

Here, we consider an example that the source has reached its power constraint after Step 1. This is based on the truth that the source always need to spend much more power at Step $1^{1}$. Therefore, we can have the following description

$$
\begin{aligned}
& \Delta P=P-\sum_{m=0}^{M-1} \tilde{p}_{m}^{(\mathrm{s})}, \\
& \Delta Q=Q-\sum_{m=0}^{M-1} \tilde{p}_{m}^{(\mathrm{r})},
\end{aligned}
$$

where $\Delta P, \Delta Q$ denotes the residual available power at the source or at the relay after Step1. Obviously, loading more bits onto the system needs the extra cost of source power (denoted by $\Delta \tilde{p}^{(\mathrm{s})}$ ) and relay power (denoted by $\Delta \tilde{p}^{(\mathrm{r})}$ ). However, the source power $\Delta P$ is not sufficient to support this requirement. Therefore, we may need to get some source power back from the loaded subcarriers. The target performance can be reached by compensating with the relay power. In order to minimize the power consumption per bit, the source power should be abstracted from the subcarrier who needs the minimum compensation of the relay power. This problem can be described as the following mathematical model.

After Step 1, each loaded subcarrier can have the following power allocation (derived from (7)-(8))

$$
\tilde{p}_{m}^{(\mathrm{r})}=\frac{1}{\left|h_{m}^{(\mathrm{rd})}\right|^{2}} \frac{\mathcal{N}_{o} \gamma^{(\mathrm{d})}\left|h_{m}^{(\mathrm{sr})}\right|^{2} \tilde{p}_{m}^{(\mathrm{s})}-\left|h_{m}^{(\mathrm{sr})}\right|^{2}\left|h_{m}^{(\mathrm{sd})}\right|^{2}\left(\tilde{p}_{m}^{(\mathrm{s})}\right)^{2}}{\left(\left|h_{m}^{(\mathrm{sr})}\right|^{2}+\left|h_{m}^{(\mathrm{sd})}\right|^{2}\right) \tilde{p}_{m}^{(\mathrm{s})}-\gamma^{(\mathrm{d})} \mathcal{N}_{o}}
$$

We can justify that $\tilde{p}_{m}^{(\mathrm{r})}$ is a positive and monotonically decreasing function of $\tilde{p}_{m}^{(\mathrm{s})}$ under the condition C3) $\tilde{p}_{m}^{(\mathrm{s})}>$ $\left(\gamma^{(\mathrm{d})} \mathcal{N}_{o}\right) /\left(\left|h_{m}^{(\mathrm{sr})}\right|^{2}+\left|h_{m}^{(\mathrm{sd})}\right|^{2}\right)$. This is the criterion for the relay to perform the power compensation. Assuming that the reduction of source power is $\delta^{(\mathrm{s})}=\Delta \tilde{p}^{(\mathrm{s})}-\Delta P$, the required power compensation from the relay should be

$$
\begin{aligned}
\delta_{m}^{(\mathrm{r})}= & \frac{\mathcal{N}_{o} \gamma^{(\mathrm{d})}\left|h_{m}^{(\mathrm{sr})}\right|^{2}\left(\tilde{p}_{m}^{(\mathrm{s})}-\delta^{(\mathrm{s})}\right)-\left|h_{m}^{(\mathrm{sr})}\right|^{2}\left|h_{m}^{(\mathrm{sd})}\right|^{2}\left(\tilde{p}_{m}^{(\mathrm{s})}-\delta^{(\mathrm{s})}\right)^{2}}{\left|h_{m}^{(\mathrm{rd})}\right|^{2}\left(\left(\left|h_{m}^{(\mathrm{sr})}\right|^{2}+\left|h_{m}^{(\mathrm{sd})}\right|^{2}\right)\left(\tilde{p}_{m}^{(\mathrm{s})}-\delta^{(\mathrm{s})}\right)-\gamma^{(\mathrm{d})} \mathcal{N}_{o}\right)} \\
& -\tilde{p}_{m}^{(\mathrm{r})} .
\end{aligned}
$$

According to the condition $\mathrm{C} 3$ ), the power reduction $\delta^{(\mathrm{s})}$ should fulfill $\delta^{(\mathrm{s})}<\tilde{p}_{m}^{(\mathrm{s})}-\left(\gamma^{(\mathrm{d})} \mathcal{N}_{o}\right) /\left(\left|h_{m}^{(\mathrm{sr})}\right|^{2}+\left|h_{m}^{(\mathrm{sd})}\right|^{2}\right)$. Subcarriers without fulfilling this condition should not be selected in the compensation process. We can calculate $\delta_{m}^{(\mathrm{r})}$ for each candidate subcarrier and obtain their minimum

$$
\delta_{\hat{m}}^{(\mathrm{r})}=\arg \min _{m} \delta_{m}^{(\mathrm{r})} .
$$

\footnotetext{
${ }^{1}$ Certainly, in some cases, the relay may reach the power constraint before the source. Although the detail mathematical formulation is not exactly identical for both examples, the idea behind is the same.
} 
Then, we can abstract $\delta^{(s)}$ source power from the $\hat{m}$ th subcarrier and compensate the performance with $\delta_{\hat{m}}^{(\mathrm{r})}$ relay power. After this process, the residual power is 0 at the source, and $\left(\Delta Q-\delta_{\hat{m}}^{(\mathrm{r})}-\Delta \tilde{p}^{(\mathrm{r})}\right)$ at the relay. The above process should be repeated until the relay power is up.

\section{Simulation Results}

Computer simulations were used to examine the number of bits/symbol and the average source symbol SNR for AFbased three-node relaying communications. A flow chart is given to provide the detailed description of the proposed BPL approach in Fig. 2. The results were obtained by averaging over 5000 independent Rayleigh channels. The linear MMSE method was employed for the channel equalization. The target SER for the bit and power loading algorithm were set up as $1 \times 10^{-3}, 1 \times 10^{-4}$, respectively. The CP-OFDM system setup was given by (the typical setup for HIPERLAN/2 in [16]): $M=64$ subcarriers, 8 samples in the CP. The OFDM block duration (exclusive $\mathrm{CP}$ ) was $3.2 \mu \mathrm{s}$. Each burst consisted of 1 OFDM blocks. The information-bearing bits were randomly generated independent and equally likely. The modulation schemes were $N$-QAM with $N=4,16,64$, respectively. The channel impulse response for each link was generated according to the indoor channel model A specified by ETSI for HIPERLAN/2 [17]. The SNR was defined as the source transmitted symbol-energy to noise ratio. With respect to various physical environments, the proposed bit and power loading algorithm was tested for the following two channelgain setups. For instance, Case I denoted the equal distance among three nodes, Case II the relay close to the source.

\begin{tabular}{llll}
\hline & SD & RD & SR \\
Case I: & 1 & 1 & 1 \\
Case II: & 1 & 1 & 10 \\
\hline
\end{tabular}

1) Experiment 1: The objective of this experiment is to evaluate the proposed sub-optimum approach for different target performance with the same power constraint at source and relay. We first plotted the average number of bits/symbol in different Cases with the target performance was given as $1 \times 10^{-3}$ in Fig. 3. It is observed that the proposed compensate approach offerers higher number of bits/symbol than the optimum approach without compensation in all SNR range for both cases, when the optimum distributed power ratio cannot be achieved. When the source symbol SNR is higher than $12 \mathrm{~dB}$, the compensate process $(\mathrm{CP})$ offers around 0.8 bits/symbol than the optimum process (OP) approach in Case I, and about 0.5 bits/symbol in Case II.

Then we set up the target performance as $1 \times 10^{-4}$. The results was plotted in Fig. 4. As the changing of the target performance, it is noticed that the average number of bits/symbol decreased in all SNR range for both cased. Higher than $12 \mathrm{~dB}$ of the source symbol SNR, the CP outperforms the 0.8 bits/symbol in Case I, and about 0.5 bits/symbol in Case II.

2) Experiment 2: The objective of this experiment is to evaluate the proposed sub-optimum approach without the same local power constraint at source and relay for target performance. We set up the source to equip as twice as the power of relay, i.e., $\mathbf{P}=2 \mathbf{Q}$. We plotted the average number of bits/symbol versus the source symbol SNR with $\mathcal{P}_{m}^{(\mathrm{d})}=1 \times 10^{-3}$ in Fig. 5. It is observed, when the source symbol SNR is higher than $12 \mathrm{~dB}$, the $\mathrm{CP}$ could improve 0.4 bits/symbol in Case I, and 0.25 bits/symbol in Case II.

We then change the target performance into $\mathcal{P}_{m}^{(\mathrm{d})}=1 \times 10^{-4}$. The results were plotted in Fig. 6. The CP offers about 0.4 bits/symbol higher than OP in Case I and 0.2 bits/symbol in Case II, when the source symbol SNR is higher than $12 \mathrm{~dB}$.

\section{CONCLUSION}

In this paper, we have investigated the RA-BPL for OFDM communication with half-duplex AF cooperative relaying. Separate power constrains were considered. It has been shown that the proposed RA-BPL algorithm could significantly maximize the number of bits/symbol. When the same distance was set up for source, relay and destination, higher number of bits/symbol achieved than the relay was placed near the source.

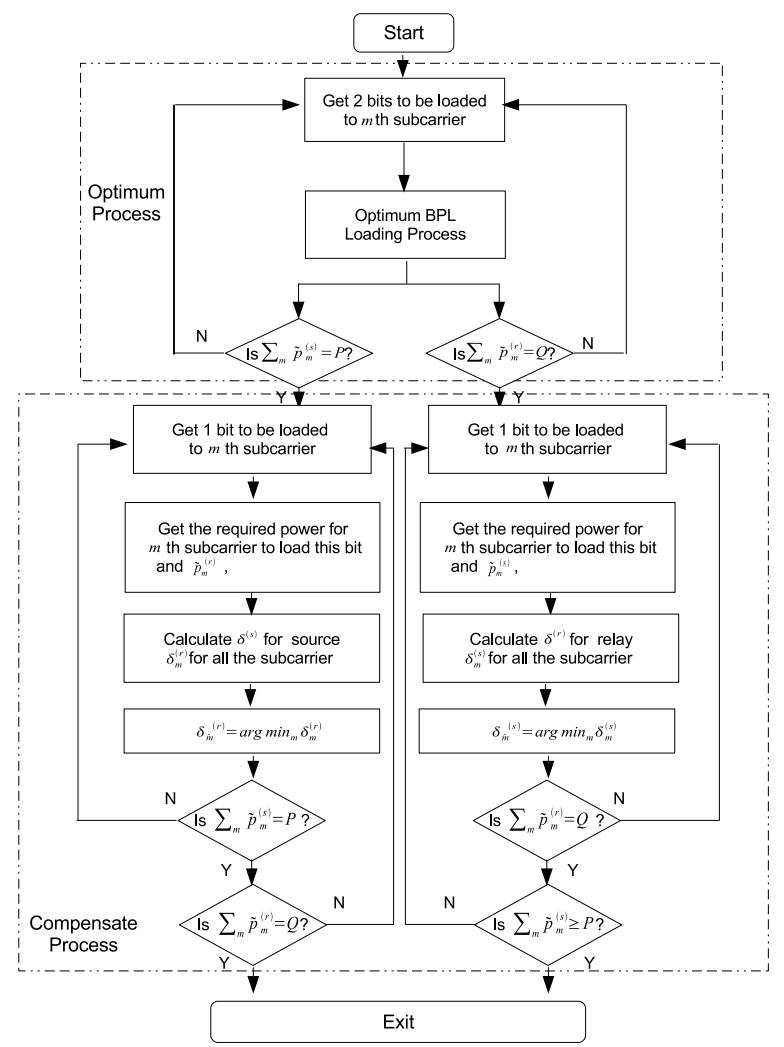

Fig. 2. Flow chart of the compensate BPL algorithm

\section{REFERENCES}

[1] E. C. van der Meulan, "Three-terminal communication channels", $A d v$. Appl. Probab., vol. 3, no. 1, pp. 120-154, Spring 1971.

[2] T. Cover and A. E. Gamal, "Capacity theorems for the relay channel", IEEE Trans. Inform. Theory, vol. 25, no. 5, pp. 572-584, Sep. 1979. 
[3] A. El. Gammal and M. Aref, "The capacity of the semi-deterministic relay channel", IEEE Trans. Inform. Theory, vol. 28, no. 3, pp. 536, May 1982.

[4] G. Kramer, M. Gastpar, and P. Gupta, "Cooperative strategies and capacity theorems for relay networks", IEEE Trans. Inform. Theory, vol. 51, no. 9, pp. 3037-3063, Sep. 2005.

[5] J. N. Laneman, D. N. C. Tse, and G. W. Wornell, "Cooperative diversity in wireless networks: effi cient protocols and outage behavior", IEEE Trans. Inform. Theory, vol. 50, no. 12, pp. 3062-3080, Dec. 2004.

[6] J. N. Laneman and G. W. Wornell, "Distributed space-time coded protocols for exploiting cooperative diversity in wireless networks", IEEE Trans. Inform. Theory, vol. 49, no. 10, pp. 2415-2525, Oct. 2003.

[7] K. Azarian, H. El Gamal, and P. Schniter, "On the achievable diversitymultiplexing tradeoff in half-duplex cooperative channels", IEEE Trans. Inform. Theory, vol. 51, no. 12, pp. 4152-4172, Dec. 2005.

[8] A. Sendomaris, E. Erkip, and B. Aazhang, "User cooperation diversity - Part I: system description”, IEEE Trans. Communs., vol. 51, no. 11, pp. 1927-1938, Nov. 2003.

[9] J. M. Cioffi, "Course notes ee379c- advanced digital communication (Chpater 4)", Course notes, University of Stanford, http://www.stanford.edu/class/ee379c, 2007.

[10] C. Y. Wong, R. S. Cheng, K. B. Letaief, and R. D. Murch, "Multiuser OFDM with adaptive subcarrier, bit and power allocation", IEEE Journal on Selected Areas in Commun., vol. 17, no. 10, pp. 1747-1758, Oct. 1999.

[11] Y. Ma, N. Yi, and R. Tafazolli, "Bit and power loading for OFDM-based three-node relaying communications", IEEE Trans. Signal Processing, Aug. 2008 (To appear).

[12] Y. Yao, X. Cai, and G. B. Giannakis, "On energy effi ciency and optimum resource allocation of relay transmission in the low-power regime", IEEE Trans. Wireless Commun., vol. 4, no. 6, pp. 2917-2927, Nov. 2005.

[13] D. Gunduz and E. Erkip, "Opportunistic cooperation by dynamic resource allocation", IEEE Trans. Wireless Commun., vol. 6, no. 4, pp. 1446-1454, Apr. 2007.

[14] K. Lee and A. Yener, "Iterative power allocation algorithms for amplify/estimate/compress-and-forward multi-band relay channels", in Proc. CISS'06, Princeton, Mar. 2006.

[15] Z. Wang and G. B. Giannakis, "Wireless multicarrier communications: Where Fourier meets Shannon", IEEE Signal Processing Magazine, vol. 17, pp. 29-48, May. 2000.

[16] ESTI, "Broadband radio access networks (BRAN); high performance radio local area networks (HIPERLAN) type 2: physical layer", in document DTS0023003, France, Dec. 1999.

[17] J. Medbo and P. Schramm, "Channel models for HIPERLAN/2 in different indoor scenarios", in BRAN 3ER1085B, Mar. 1998.

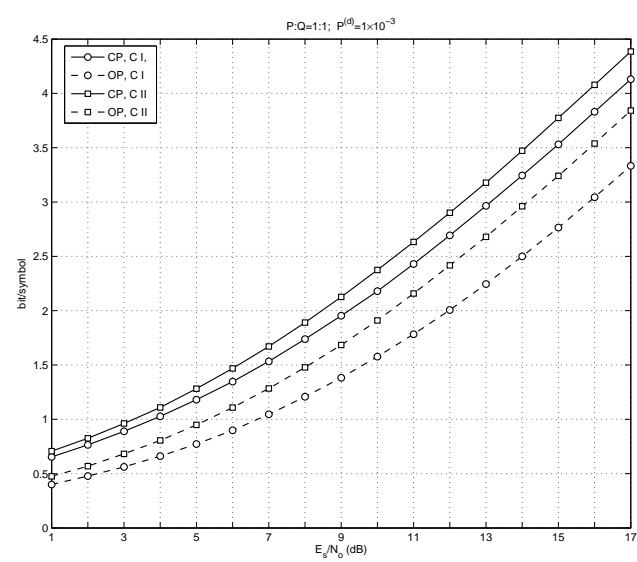

Fig. 3. The average number of bits/symbol versus source symbol SNR; $P: Q=1: 1 ; \mathcal{P}^{(\mathrm{d})}=1 \times 10^{-3}$

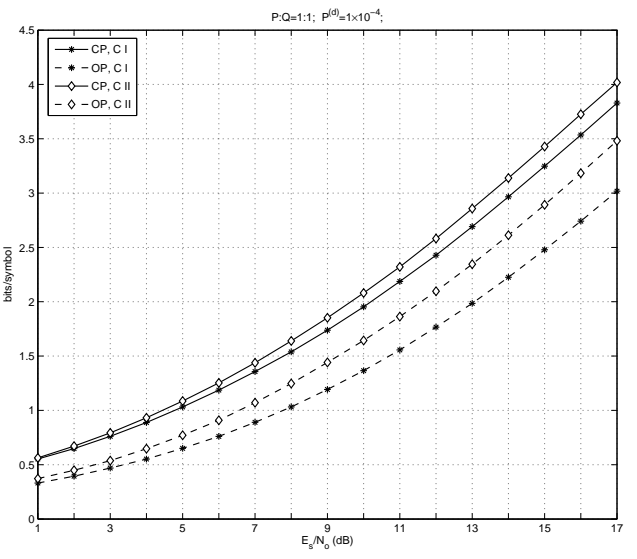

Fig. 4. The average number of bits/symbol versus source symbol SNR; $P: Q=1: 1 ; \mathcal{P}^{(\mathrm{d})}=1 \times 10^{-4}$

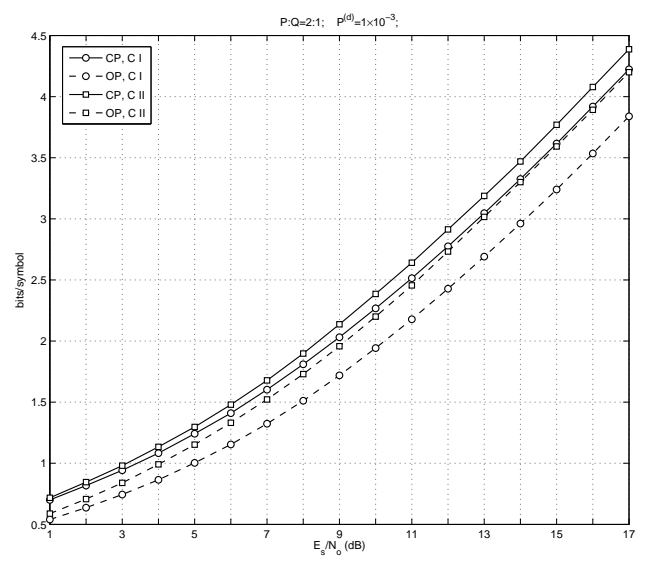

Fig. 5. The average number of bits/symbol versus source symbol SNR; $P: Q=2: 1 ; \mathcal{P}^{(\mathrm{d})}=1 \times 10^{-3}$

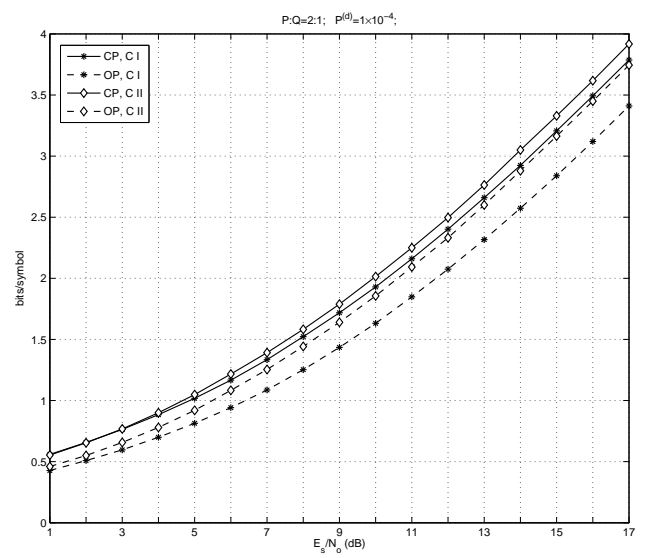

Fig. 6. The average number of bits/symbol versus source symbol SNR; $P: Q=2: 1 ; \mathcal{P}^{(\mathrm{d})}=1 \times 10^{-4}$ 